\title{
Queixas fonoaudiológicas e verificação da fala de indivíduos com diagnóstico de ardência bucal e xerostomia
}

\author{
Complaints to oral functions and verification of the speech of \\ individuals diagnosed with burning mouth and xerostomia
}

\author{
Silvana da Gama Pastana1', Marília Heffer Cantisano², Esther Mandelbaum Gonçalves Bianchini
}

\begin{abstract}
RESUMO
Objetivo: Investigar as queixas das funções orais em presença dos sintomas de ardência e secura bucal e analisar as alterações da fala em seu aspecto articulatório. Métodos: Foram avaliados 66 indivíduos com idade entre 30 e 78 anos, divididos em três grupos: grupo ardência bucal, grupo xerostomia e grupo sem sintomas bucais. Foram realizadas entrevistas, exame clínico da cavidade oral e gravação da fala, com utilização de fichário evocativo. Resultados: A característica comum nos dois primeiros grupos foi a presença do sintoma de secura bucal. Na localização dos sintomas, o grupo xerostomia apresentou maior quantidade de estruturas afetadas pelo sintoma. As queixas mais referidas por esse grupo foram cansaço e força na fala e força e engasgos à deglutição. A queixa de força foi significativa, na comparação com grupo de ardência bucal, com aumento do sintoma provocado pela função de fala. Dos sujeitos que se queixaram de boca seca, como sintoma principal, ou associado, a maioria apresentou ruídos durante a fala. O grupo xerostomia apresentou maior ocorrência desse ruído. Não foram evidenciadas alterações fonéticas nos grupos de sintomas bucais. Conclusão: Das queixas envolvendo as funções orais, falar e deglutir com força foram as mais referidas pelos indivíduos do grupo xerostomia. Observou-se a presença de estalidos na fala da maioria dos sujeitos com o sintoma de secura bucal. Apesar das sintomatologias apresentadas e do número de estruturas orais afetadas, não houve evidência de alteração fonética nos indivíduos com sintomas bucais.
\end{abstract}

Descritores: Síndrome da Ardência Bucal; Xerostomia; Fonética; Fala; Medicina Bucal

\begin{abstract}
Purpose: To investigate complaints related to oral functions in the presence of burning mouth and dry mouth, and analyze changes in the manner of articulation of speech. Methods: There were 66 participants, age range 30-78 years, arranged in three groups: burning mouth group, xerostomia group, and group of individuals without oral symptoms. Interviews, as well as a clinical exam of the oral cavity and the recording of the subjects' speech based on a pre-set list of words, were carried out. Results: Dry mouth was found as a common characteristic for the first two groups. Regarding symptom localization, the xerostomia group described a larger amount of structures affected by the symptom. The complaints that were most frequently reported by this group were tiredness and struggle to speak, and struggle and choke during deglutition. The struggle complaint was significant in comparison with the burning mouth group, and the symptom was aggravated in presence of speech. Most of the subjects reporting dry mouth either as the main symptom or as an associated symptom gave off clicks during speech. A higher incidence of those clicks was found in the xerostomia group. No evidence of phonetic changes were found in the symptomatic groups. Conclusion: Regarding the complaints involving the oral functions, struggle speaking and swallowing were the most frequently reported by the subjects in the xerostomia group. Clicks were found in the speech of the majority of the subjects with dry mouth. Despite the symptomatology identified and the number of affected oral structures. Despite the symptomatology and the number of affected oral structures, no evidence of phonetic changes in the individuals with oral symptoms.
\end{abstract}

Keywords: Burning Mouth Syndrome; Xerostomia; Phonetics; Speech; Oral Medicine

Trabalho realizado na Universidade Veiga de Almeida - UVA - Rio de Janeiro (RJ), Brasil e na Universidade do Estado do Rio de Janeiro - UERJ - Rio de Janeiro (RJ), Brasil.

(1) Faculdade de Odontologia, Universidade do Estado do Rio de Janeiro - UERJ - Rio de Janeiro (RJ), Brasil; Programa de Pós-Graduação (Mestrado Profissional) em Fonoaudiologia, Universidade Veiga de Almeida - UVA - Rio de Janeiro (RJ), Brasil.

(2) Faculdade de Odontologia, Departamento de Diagnóstico e Cirurgia em Odontologia, Universidade do Estado do Rio de Janeiro - UERJ - Rio de Janeiro (RJ), Brasil.

(3) Programa de Pós-graduação (Mestrado Profissional) em Fonoaudiologia, Universidade Veiga de Almeida - UVA - Rio de Janeiro (RJ), Brasil.

Conflito de interesses: Não

Contribuição dos autores: SGP pesquisador principal, elaboração da pesquisa, elaboração do cronograma, levantamento da literatura, coleta e análise dos dados, redação do artigo, submissão e trâmites do artigo; $M H C$ coorientadora, elaboração da pesquisa, elaboração do cronograma, análise dos dados, correção da redação do artigo, aprovação da versão final; $E M G B$ orientadora, elaboração da pesquisa, elaboração do cronograma, análise dos dados, correção da redação do artigo, aprovação da versão final.

Endereço para correspondência: Silvana da Gama Pastana. Faculdade de Odontologia, Universidade do Estado do Rio de Janeiro, Setor de Fonoaudiologia, Edifício Paulo de Carvalho. R. 28 de Setembro, 157, Vila Isabel, Rio de Janeiro (RJ), Brasil, CEP: 20551-030. E-mail: sgpastana@ig.com.br

Recebido em: 18/1/2013; Aceito em: 4/9/2013 


\section{INTRODUÇÃO}

A síndrome da Ardência Bucal (SAB) é uma doença que se caracteriza por queimação e/ou dor constante na boca, sem que esteja associada às lesões em cavidade oral. A ocorrência da SAB é maior em mulheres de meia-idade e idosos ${ }^{(1,5)}$. A intensidade da ardência, ou queimação, pode ser de grau variável, sendo intensificada no decorrer do dia ${ }^{(4,6)}$. A queimação ocorre frequentemente em mais de uma área ${ }^{(2,4)}$ e a língua é citada como a estrutura mais comprometida ${ }^{(2-7)}$, podendo ainda, atingir outras regiões da cavidade oral, como lábios, palato, gengiva e mucosa jugal $^{(3-5)} \mathrm{e}$, com menor frequência, o assoalho da boca ${ }^{(3)}$ e a orofaringe ${ }^{(2)}$. O termo "síndrome" é utilizado pelo fato da SAB ser descrita em associação simultânea da ardência com outros sintomas subjetivos, como xerostomia e disgeusia ${ }^{(2,4,7,8)}$. Por sua condição enigmática, a etiologia é apontada como multifatorial $^{(5)}$.

As queixas referentes às funções orais são descritas pelo agravamento da sintomatologia no ato de falar e no consumo de comidas quentes ${ }^{(9)}$, na ingestão de alimentos ácidos ${ }^{(10)} \mathrm{e}$ picantes $^{(7,11)}$, na melhora ou desaparecimento do sintoma com a ingestão de bebida e comida ${ }^{(6,11)}$, no alívio do sintoma ao ingerir comidas frias ${ }^{(10)}$. Os indivíduos relatam, ainda, que a ingestão de bebidas ou alimentos com temperaturas extremas podem agravar ou aliviar os sintomas ${ }^{(2)}$.

A saliva, por suas múltiplas funções, diretamente relacionadas com a quantidade do fluxo e de seus componentes específicos, possui papel fundamental na manutenção da saúde do indivíduo. Quaisquer alterações que venham a afetar sua quantidade e qualidade poderão, em menor ou maior grau de severidade, levar a consequências diversas e interferir em toda a homeostase do organismo ${ }^{(8)}$. O papel que a saliva desempenha na cavidade oral vai desde a lubrificação da mucosa até as funções gastrointestinais ${ }^{(12)}$. A saliva exerce função de defesa pela depuração bacteriana e limpeza de detritos, umidificação e diluição de alimentos, favorecendo a formação do bolo alimentar, facilitando a mastigação, a deglutição e a fala ${ }^{(13)}$.

Um dos sintomas relacionados à alterações na saliva é a xerostomia, manifestação clínica mais comum das queixas bucais, por sua associação com diversos fatores ${ }^{(13-15)}$. É descrita como uma sensação subjetiva de boca seca, não refletindo, necessariamente, uma condição de hipossalivação, que seria a diminuição do fluxo salivar ${ }^{(16)}$. Dentre as várias causas, a Síndrome de Sjögren é a doença sistêmica que mais compromete a saliva. Sua condição autoimune provoca a inflamação crônica das glândulas salivares, com ocorrência de desordem nos componentes químicos, provocando alteração quantitativa e qualitativa da saliva ${ }^{(17,18)}$, sendo a xerostomia a principal manifestação dessa síndrome ${ }^{(19)}$. A secura bucal pode atingir a mucosa oral como um todo ${ }^{(20)}$, porém, os sujeitos podem referir acometimento do sintoma em estruturas como lábios ${ }^{(21)} \mathrm{e}$ orofaringe ${ }^{(16,22)}$. A ocorrência do sintoma pode ser contínua ${ }^{(23)}$, com períodos de desconforto considerável ${ }^{(20)}$.
A xerostomia é constantemente referida em associação à $\operatorname{ardência~bucal~}^{(2,15,23,24)}$, assim como à alteração do paladar ${ }^{(4,8)}$. A presença de distúrbios do paladar pode aumentar o desconforto oral e prejudicar o apetite e a ingestão de alimentos, influenciando na qualidade de vida do indivíduo ${ }^{(24)}$. Os sujeitos relatam, ainda, que, para tentar aliviar o desconforto oral, fazem uso de estratégias e/ou medicamentos para minimizar sua sintomato$\operatorname{logia}{ }^{(19,23)}$. Devido à relação da xerostomia com vários fatores $\mathrm{e}$ frequente associação a outros sintomas bucais, as terapêuticas utilizadas têm efeitos paliativos e visam reduzir o desconforto oral, a fim de reduzir as diversas queixas dos indivíduos com sintoma de boca seca ${ }^{(14,18,19,23)}$.

Muitos estudos fazem referência ao impacto que o sintoma de secura bucal provoca nas funções de fala, mastigação e deglutição $0^{(14-19,23-26)}$, podendo comprometer também a voz ${ }^{(27)}$. As queixas relacionadas à xerostomia são, geralmente, identificadas por meio de questionários ${ }^{(15-17,19,23,25)}$. Alguns estudos procuram investigar, de forma objetiva, o comprometimento das funções orais relacionadas ao sintoma de boca seca e/ou hipossalivação ${ }^{(17,28)}$, mas para a função de fala, poucos estudos foram encontrados ${ }^{(29,30)}$.

O objetivo deste estudo foi descrever as características de interesse fonoaudiológico dos indivíduos com diagnóstico de ardência bucal e xerostomia, identificar possíveis interferências nas funções orais e o impacto desses sintomas na articulação da fala.

\section{MÉTODOS}

Esta pesquisa foi aprovada pelo Comitê de Ética em Pesquisa do Hospital Universitário Pedro Ernesto, da Universidade do Estado do Rio de Janeiro (UERJ), sob o n ${ }^{\circ} 2856 / 2011$, sendo considerada sem risco e com necessidade de assinatura do Termo de Consentimento Livre e Esclarecido por todos os participantes. Fizeram parte desta pesquisa, pacientes atendidos na Policlínica Piquet Carneiro, que foram encaminhados à avaliação fonoaudiológica pela Clínica de Estomatologia da UERJ, com diagnóstico de ardência bucal e de xerostomia. O diagnóstico odontológico foi realizado anteriormente por uma odontóloga, especialista em patologia bucal. Por tratar-se de sintomatologia, o diagnóstico foi feito por meio da observação dos sinais clínicos, com base nos dados da anamnese, avaliação clínica e inspeção da cavidade oral. Também foram avaliados indivíduos que não apresentaram queixas de ardência e secura bucal, para composição do Grupo controle.

A amostra foi constituída por 66 sujeitos, sendo 62 do gênero feminino e quatro do gênero masculino, com idade entre 30 e 78 anos, assim distribuídos: G1 = Grupo de 22 indivíduos com diagnóstico de ardência bucal (20 do gênero feminino e dois do gênero masculino), faixa etária de 44 a 78 anos, média de idade de 60,4 anos; G2 = Grupo de 22 indivíduos do gênero feminino, com diagnóstico de xerostomia, faixa etária de 34 a 70 anos, média de idade de 58,7 anos; G3 = Grupo de 22 
indivíduos sem queixas de ardência e/ou secura bucal (20 do gênero feminino e dois do gênero masculino), faixa etária de 30 a 69 anos, média de idade de 50,2 anos.

Foram adotados os seguintes critérios de exclusão: deficiências neurológicas e/ou cognitivas, doenças neurológicas congênitas, adquiridas ou evolutivas; deficiência auditiva, disfluência; deformidades dentofaciais ou distúrbios da articulação temporomandibular; qualquer tipo de lesão na cavidade oral e uso de aparelhos ortodônticos ou piercing nas estruturas orais.

Os indivíduos foram entrevistados e avaliados pela fonoaudióloga autora da pesquisa. Todos foram atendidos em ambiente fechado, individualmente, com horários preestabelecidos. Para cada indivíduo, foi realizada uma entrevista contendo dados de identificação (nome, idade, gênero) e diagnóstico odontológico. O histórico clínico da sintomatologia incluiu: tempo de acometimento da doença; sintomas associados; local afetado; intensidade do sintoma, verificada por meio da escala visual analógica (EVA), para a qual foram atribuídos escores que variaram de 1 a 10 , considerados como intensidade mínima e máxima. Para identificar essa intensidade, os indivíduos deveriam atribuir um valor ao seu sintoma, respondendo à pergunta do avaliador "Que nota o(a) $\operatorname{Sr}$ (a) daria à intensidade desse sintoma?" e também fornecer as seguintes informações: se o sintoma era contínuo; se havia ocorrência de período de intensidade do sintoma; que estratégias utilizavam para amenizar o sintoma; tipos de queixas orais provocadas pelo sintoma; se o sintoma aumentava ou diminuía na função oral; se ocorria modificação da função provocada pelo sintoma.

Em seguida, foram submetidos ao exame da cavidade oral, a fim de se inspecionar a integridade de todas as estruturas envolvidas na função articulatória da fala. No protocolo de inspeção oral, constavam as condições das seguintes estruturas: condição dentária, tipo de mordida, características da língua, lábios, bochechas e amígdalas. Após explicação sobre a gravação da fala, o headset com microfone (Bright@ 01409) foi colocado na cabeça do paciente, que se encontrava na posição ereta e de frente para o computador, para favorecer a visualização das gravuras. A gravação de áudio (Computador Netbook Sony Vaio®, Windows 7®) e a filmagem (Câmera/ Filmadora Sony ${ }^{\circledR}$ DSC-W320) ocorreram de forma simultânea. A gravação constou de emissão do nome, idade e nomeação das figuras do fichário evocativo com 87 figuras, contendo todos os sons do Português brasileiro. Os registros de fala foram foneticamente transcritos e analisados.

Como forma de facilitar a mensuração na verificação da intensidade dos sintomas, com uso da escala visual analógica (EVA), foram atribuídos escores que variaram de 1 a 10 . Os escores foram agrupados em três graus de intensidade: leve (1-3), moderada (4-7) e severa (8-10).

Tanto o questionário quanto os protocolos de inspeção oral e fala, utilizados na entrevista e na avaliação, foram elaborados especificamente para este estudo pelas duas fonoaudiólogas autoras da pesquisa, do Programa de Pós-Graduação Mestrado profissional em Fonoaudiologia da Universidade Veiga de Almeida (UVA).

Os dados foram apresentados na forma de tabelas, expressos pela frequência (n) e percentual (\%), para dados categóricos nominais e ordinais e, média, desvio padrão, mediana, amplitude interquartílica (AIQ), mínimo e máximo, para dados quantitativos. Com o objetivo de verificar a existência de diferença significativa para as variáveis testadas (tempo de doença; número de sintomas associados; número de estruturas afetadas; intensidade dos sintomas; período de intensidade; sintoma contínuo; uso e número de estratégias; tipos de queixas nas funções orais; mudanças percebidas e número de sujeitos com estalidos) entre os grupos G1 (ardência bucal), G2 (xerostomia) e G3 (controle), foram aplicados o teste $\chi^{2}$ Qui quadrado, ou exato de Fisher, para comparações de dados categóricos e, para comparação de dados numéricos, foram aplicadas a ANOVA one-way (idade) e a ANOVA não paramétrica de Kruskall-Wallis (número de estalidos). Os testes de comparações múltiplas de Tukey e de Dunn (não paramétrico) foram utilizados para identificar quais os grupos que diferiram significativamente entre si, ao nível de 5\%.

Foi utilizado método não paramétrico, pois algumas variáveis não apresentaram distribuição normal (distribuição Gaussiana), devido à grande dispersão e rejeição da hipótese de normalidade segundo o teste de Kolmogorov-Smirnov. O critério de determinação de significância adotado foi o nível de 5\%. A análise estatística foi processada pelo software estatístico SAS ${ }^{\circledR}$ System, versão 6.11 (SAS Institute, Inc., Cary, North Carolina).

\section{RESULTADOS}

Nos grupos G1 e G2, a variação do tempo da doença foi de um ano a mais de 10 anos. Quanto à distribuição do número de sintomas associados, houve diferença entre os grupos. O G1 apresentou maior quantidade de sujeitos com dois sintomas associados, enquanto que o G2 apresentou, em sua maioria, um único sintoma associado, por indivíduo. Os grupos não diferiram quanto ao número de estruturas afetadas. Quanto à intensidade dos sintomas, mensurada por meio de Escala Analógica Visual (EVA), observou-se que os grupos sintomáticos apresentaram intensidade moderada, não havendo diferença entre os grupos. Verificou-se semelhança entre os grupos, no que diz respeito aos períodos de intensidade e ocorrência do sintoma. Os dois grupos referiram os sintomas como contínuos e também que intensificam-se em determinados períodos. Quanto ao uso de estratégias para minimizar os sintomas, constatou-se percentuais elevados em ambos grupos. Observouse que, na distribuição dos números de estratégias, a maioria dos sujeitos, tanto do G1 quanto do G2, utiliza, pelo menos, um tipo de estratégia para alívio do sintoma, sem diferença entre os grupos (Tabela 1).

$\mathrm{Na}$ identificação dos sintomas associados, a ardência 
Tabela 1. Comparação do histórico clínico nos dois grupos de sintomas bucais

\begin{tabular}{|c|c|c|c|c|c|}
\hline \multirow[t]{2}{*}{ Histórico clínico dos sintomas } & \multicolumn{2}{|c|}{$\begin{array}{c}\mathrm{G} 1 \\
(\mathrm{n}=22)\end{array}$} & \multicolumn{2}{|c|}{$\begin{array}{c}G 2 \\
(n=22)\end{array}$} & \multirow[t]{2}{*}{ Valor de $p$} \\
\hline & $\mathrm{n}$ & $\%$ & $\mathrm{n}$ & $\%$ & \\
\hline \multicolumn{6}{|l|}{ Tempo de doença } \\
\hline 1 a 5 anos & 16 & 72,7 & 10 & 45,5 & 0,20 \\
\hline 5 a 10 anos & 4 & 18,2 & 9 & 40,9 & \\
\hline$>10$ anos & 2 & 9,1 & 3 & 13,6 & \\
\hline \multicolumn{6}{|l|}{$\mathrm{N}^{\circ}$ de sintomas associados } \\
\hline 0 & 3 & 13,6 & 6 & 27,3 & $0,039^{*}$ \\
\hline 1 & 5 & 22,7 & 10 & 45,5 & \\
\hline 2 & 12 & 54,5 & 3 & 13,6 & \\
\hline 3 ou 4 & 2 & 9,1 & 3 & 13,6 & \\
\hline \multicolumn{6}{|l|}{$\mathrm{N}^{\circ}$ de estruturas afetadas } \\
\hline 1 a 2 & 15 & 68,2 & 10 & 45,5 & 0,081 \\
\hline 3 a 4 & 6 & 27,3 & 5 & 22,7 & \\
\hline$\geq 5$ & 1 & 4,5 & 7 & 31,8 & \\
\hline \multicolumn{6}{|l|}{ Intensidade dos sintomas } \\
\hline leve (1 a 3) & 2 & 9,1 & 1 & 4,5 & 0,63 \\
\hline moderada (4 a 7) & 14 & 63,6 & 12 & 54,5 & \\
\hline severa (8 a 10) & 6 & 27,3 & 9 & 40,9 & \\
\hline Ocorrência de período de intensidade & 17 & 77,3 & 17 & 77,3 & 1 \\
\hline Ocorrência do sintoma contínuo & 14 & 63,6 & 14 & 63,6 & 1 \\
\hline Uso de estratégia para minimizar os sintomas & 18 & 81,8 & 16 & 72,7 & 0,47 \\
\hline \multicolumn{6}{|l|}{$\mathrm{N}^{\circ}$ de estratégias para minimizar os sintomas } \\
\hline nenhuma & 4 & 18,2 & 3 & 13,6 & 0,37 \\
\hline 1 & 9 & 40,9 & 11 & 50,0 & \\
\hline 2 & 6 & 27,3 & 8 & 36,4 & \\
\hline 3 & 3 & 13,6 & 0 & 0 & \\
\hline
\end{tabular}

*Valores significativos $(p<0,05)$ - teste de $\chi^{2}$ ou exato de Fisher

Legenda: G1 = Grupo ardência; G2 = Grupo xerostomia

apareceu com 45,5\% ( $\mathrm{n}=10)$ no grupo xerostomia e a secura, com 72,7\% (n=16) no grupo ardência bucal. As alterações de olfato e de paladar apareceram com o mesmo percentual em ambos os grupos, $18,2 \%(n=4)$ e $45,5 \%(n=10)$, respectivamente. O percentual do sintoma de dor foi de $22,7 \%(n=5)$ no grupo ardência bucal e 9,1\% (n=2) no grupo xerostomia.

Quanto à identificação das estruturas afetadas pelos sintomas de ardência e secura bucal, a língua foi mencionada como o local mais atingido, em ambos os grupos, o mesmo ocorrendo com as estruturas gengiva e palato. Com relação às estruturas lábios, mucosa jugal, orofaringe e assoalho bucal, verificou-se diferenças significativas do número de sujeitos que referiram essas estruturas como afetadas pelo sintoma de secura bucal, no grupo xerostomia, em comparação ao grupo ardência bucal. Lábios e orofaringe também aparecem com alta porcentagem no grupo xerostomia (Figura 1).

As estratégias utilizadas como forma de minimizar os sintomas bucais, e que mostraram diferenças, foram os alimentos

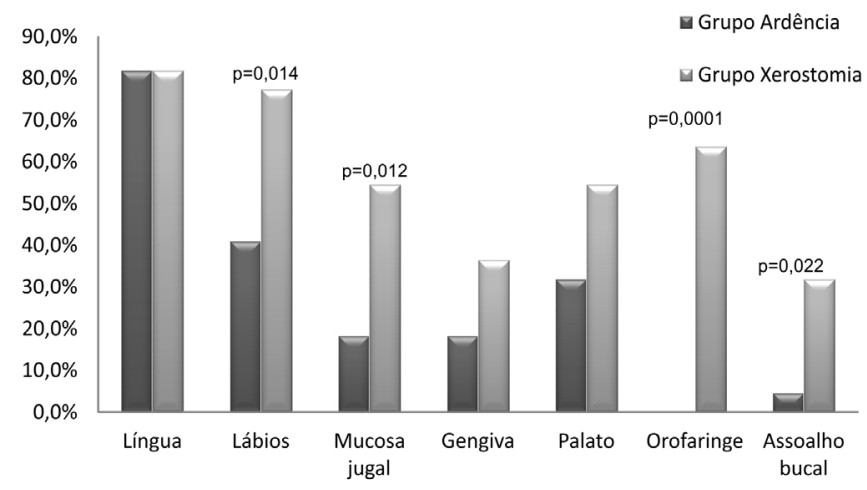

Teste de $\chi^{2}$ ou exato de Fisher $(p<0,05)$

Figura 1. Identificação das estruturas orais afetadas pelos sintomas de ardência e secura nos grupos de sintomas bucais

no G1 e os medicamentos no G2. Quanto à variável bebidas, verificou-se que, apesar do G2 ter apresentado maior porcentagem de indivíduos que utilizam essa estratégia buscando alívio, 
não foi observada diferença, quando comparado ao G1. Outras estratégias, como gelo, bochecho, distração, doces e parar de falar foram referidas e, apesar de aparecerem com maior diversidade no G1, não apresentaram diferenças na comparação com o G2 (Figura 2).

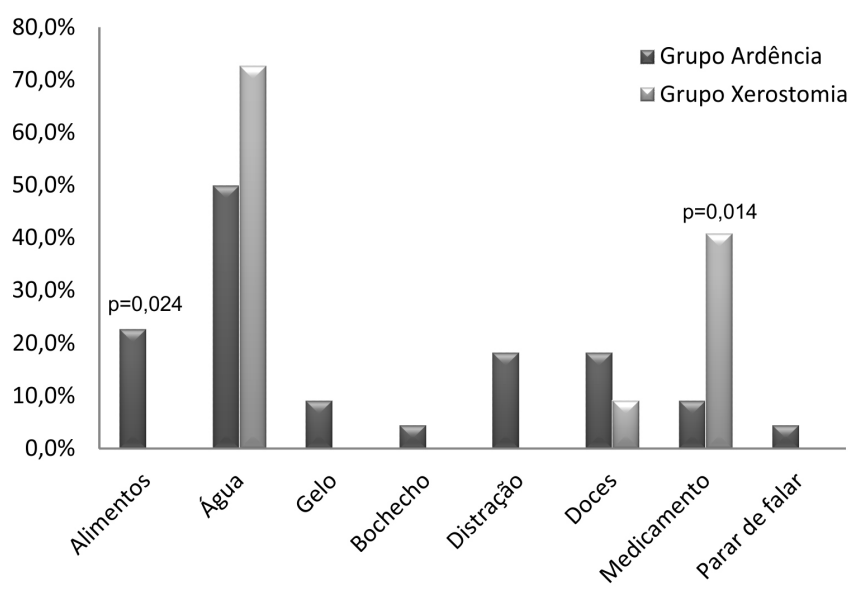

Teste de $\chi^{2}$ ou exato de Fisher $(p<0,05)$

Figura 2. Identificação das estratégias utilizadas para minimizar o desconforto oral nos grupos de sintomas bucais

Com relação às queixas nas funções orais, verificou-se que, na comparação geral dos resultados percentuais, o grupo xerostomia referiu mais queixas que o grupo ardência bucal. Observou-se, ainda, que, embora a queixa de cansaço durante a fala tenha apresentado maior porcentagem no grupo xerostomia, não houve diferença entre os grupos. Quanto à queixa do uso de força, verificou-se que o $\mathrm{G} 2$ referiu essa condição tanto na fala, quanto na deglutição, com diferenças em relação ao G1. A queixa de engasgo foi mais citada no grupo xerostomia, não observando-se diferença, no entanto, quando comparada ao grupo ardência bucal (Tabela 2).

Com relação à ocorrência de mudanças nos sintomas bucais, provocadas pelas funções orais, verificou-se, no G2, que a função de fala aumenta o sintoma de secura, com diferença quando comparada ao grupo de ardência bucal. Quanto ao efeito da função de mastigação sobre os dois sintomas pesquisados, constatou-se baixa ocorrência de sujeitos que referiram aumento dos sintomas após essa função. Observouse semelhança entre os grupos G1 e G2, no que diz respeito às modificações das funções orais produzidas pelos sintomas bucais. Quanto à fala, apesar de 27,3\% dos sujeitos com ardência bucal terem referido modificação, somente $4,5 \%$ relataram fazer uso da estratégia de parar de falar para alívio do sintoma (Figura 2) (Tabela 3).

Quanto às alterações de fala, houve baixa ocorrência de alteração fonética entre os grupos de sintomas bucais. Contudo, a metodologia utilizada no presente estudo possibilitou a observação da dinâmica articulatória e identificação de um ruído específico, como um "clique" acompanhando a fala de muitos desses sujeitos. Esses cliques foram identificados como estalidos, observados entre as sequências articulatórias, ou fazendo parte do discurso dos indivíduos com sintoma de secura bucal, independente do grupo a que pertenciam (Tabelas 4 e 5).

\section{DISCUSSÃO}

Na caracterização da amostra, houve concordância com a literatura que refere que a maioria dos pacientes com queixas de ardência bucal ${ }^{(1-5)}$ e de secura bucal ${ }^{(15,19,24,29)}$ são do gênero feminino. $\mathrm{O}$ grupo controle foi pareado quanto ao gênero, buscando-se evitar qualquer viés que a variável pudesse definir. Com relação à idade, mesmo não tendo sido controlada, por tratar-se de pacientes em sequência, não foram observadas diferenças entre os grupos de sintomas bucais. Apenas o G3 foi pareado quanto a essa variável. Esse dado pode mostrar que tanto a SAB quanto secura bucal parecem afetar, principalmente, a população com idade em torno de $50 \operatorname{anos}^{(1,15,24)}$.

Tabela 2. Distribuição e identificação das queixas nas funções orais dos grupos de ardência bucal e xerostomia

\begin{tabular}{|c|c|c|c|c|c|c|}
\hline \multirow{3}{*}{ Funções orais } & \multirow{3}{*}{ Queixas } & \multicolumn{2}{|c|}{$\begin{array}{c}\text { G1 } \\
(n=22)\end{array}$} & \multicolumn{2}{|c|}{$\begin{array}{c}\mathrm{G} 2 \\
(\mathrm{n}=22)\end{array}$} & \multirow{3}{*}{ Valor de $p$} \\
\hline & & \multicolumn{2}{|c|}{ Frequência } & \multicolumn{2}{|c|}{ Frequência } & \\
\hline & & $\mathrm{n}$ & $\%$ & $\mathrm{n}$ & $\%$ & \\
\hline \multirow[t]{3}{*}{ Fala } & Cansaço & 6 & 27,3 & 12 & 54,5 & 0,066 \\
\hline & Força & 0 & 0 & 5 & 22,7 & $0,024^{*}$ \\
\hline & Ruído & 0 & 0 & 1 & 4,5 & 0,50 \\
\hline \multirow[t]{3}{*}{ Mastigação } & Cansaço & 3 & 13,6 & 2 & 9,1 & 0,50 \\
\hline & Força & 0 & 0 & 2 & 9,1 & 0,24 \\
\hline & Ruído & 0 & 0 & 2 & 9,1 & 0,24 \\
\hline \multirow[t]{3}{*}{ Deglutição } & Força & 0 & 0 & 7 & 31,8 & $0,004^{*}$ \\
\hline & Ruído & 0 & 0 & 0 & 0 & NSA \\
\hline & Engasgo & 2 & 9,1 & 5 & 22,7 & 0,20 \\
\hline
\end{tabular}

*Valores significativos $(p<0,05)$ - teste de $\chi^{2}$ ou exato de Fisher

Legenda: $\mathrm{G} 1$ = Grupo ardência; G2 = Grupo xerostomia 
Tabela 3. Identificação das mudanças percebidas pelos sujeitos dos grupos de ardência bucal e xerostomia

\begin{tabular}{|c|c|c|c|c|c|}
\hline \multirow{3}{*}{ Mudanças percebidas } & \multirow{2}{*}{\multicolumn{2}{|c|}{$\begin{array}{c}\begin{array}{c}\mathrm{G} 1 \\
(\mathrm{n}=22)\end{array} \\
\text { Frequência }\end{array}$}} & \multirow{2}{*}{\multicolumn{2}{|c|}{$\begin{array}{c}\begin{array}{c}\mathrm{G} 2 \\
(\mathrm{n}=22)\end{array} \\
\text { Frequência }\end{array}$}} & \multirow{3}{*}{ Valor de $\mathrm{p}$} \\
\hline & & & & & \\
\hline & $\mathrm{n}$ & $\%$ & $\mathrm{n}$ & $\%$ & \\
\hline A função fala aumenta o sintoma & 7 & 31,8 & 20 & 90,9 & $<0,0001^{*}$ \\
\hline A função mastigação aumenta o sintoma & 2 & 9,1 & 2 & 9,1 & 0,70 \\
\hline O sintoma modifica a função fala & 6 & 27,3 & 3 & 13,6 & 0,22 \\
\hline O sintoma modifica a função mastigação & 2 & 9,1 & 2 & 9,1 & 0,70 \\
\hline O sintoma modifica a função deglutição & 1 & 4,5 & 2 & 9,1 & 0,50 \\
\hline
\end{tabular}

*Valores significativos $(p<0,05)$ - teste de $\chi^{2}$ ou exato de Fisher

Legenda: G1 = Grupo ardência; G2 = Grupo xerostomia

Tabela 4. Alterações fonéticas e de estalidos nos grupos estudados

\begin{tabular}{lcccc}
\hline Fala & $\begin{array}{c}\mathrm{G} 1 \\
(\mathrm{n}=22)\end{array}$ & $\begin{array}{c}\mathrm{G} 2 \\
(\mathrm{n}=22)\end{array}$ & $\begin{array}{c}\mathrm{G} 3 \\
(\mathrm{n}=22)\end{array}$ & Valor de $\mathrm{p}$ \\
\hline Alterações fonéticas & $(0-3)$ & $(0-1)$ & 0 & $\mathrm{NSA}$ \\
Estalidos & $4 \pm 10(0-22)$ & $13,5 \pm 15,3(0-62)$ & $0 \pm 2(0-34)$ & $<0,0001^{*}$ \\
\hline
\end{tabular}

${ }^{*} \mathrm{O}$ número de estalidos foi expresso pela mediana $\pm \mathrm{AIQ}$ (mínimo - máximo) e comparada pela ANOVA de Kruskal-Wallis $(p<0,0001)$

Legenda: G1 = Grupo ardência; G2 = Grupo xerostomia; G3 = Grupo controle; NSA = não se aplica

Tabela 5. Número de sujeitos com estalidos na fala na presença ou ausência do sintoma de secura bucal

\begin{tabular}{|c|c|c|c|c|c|}
\hline \multirow[t]{2}{*}{ Função x sintoma } & \multicolumn{2}{|c|}{$\begin{array}{l}\text { Com secura } \\
(n=38)\end{array}$} & \multicolumn{2}{|c|}{$\begin{array}{c}\text { Sem secura } \\
(n=6)\end{array}$} & \multirow[t]{2}{*}{ Valor de $\mathrm{p}$} \\
\hline & $\mathrm{n}$ & $\%$ & $\mathrm{n}$ & $\%$ & \\
\hline Fala associada ao estalido & 31 & 81,6 & 1 & 16,7 & $0,003^{*}$ \\
\hline
\end{tabular}

*Valores significativos $(p<0,05)$ - teste exato de Fisher

No que diz respeito ao histórico clínico, observou-se que os grupos de sintomas bucais demonstraram semelhanças em vários aspectos, o que concorda com a literatura pesquisa$\mathrm{da}^{(2,4-8,11,16,22,23)}$. Porém, alguns estudos identificaram variações, principalmente com relação à intensidade do sintoma ${ }^{(4,22,24)}$. Por tratar-se de sintomatologia em que a sensação tem caráter subjetivo, pode-se esperar a ocorrência de variações relacionadas a esse aspecto.

Este estudo confirmou o uso de estratégias para minimizar os sintomas ${ }^{(6,11,19,23)}$, dado que demonstra que os sujeitos desenvolvem mecanismos de alivio como forma de minimizar sua sintomatologia.

Houve confirmação, também, de sintomas associados, pelos dois grupos, visto que, o sintoma principal de um grupo aparece como sintoma associado no outro e vice-versa ${ }^{(4,7,8,15,23,24)}$. A diversidade de sintomas encontrados e associados aos sintomas principais de ardência e secura bucal, provavelmente dificulta o esclarecimento da causa e, por consequência, resulta em tratamentos diversos ${ }^{(14,18,19,23)}$. Com relação ao sintoma de dor, estudos referem a SAB como condição associada à dor crônica ${ }^{(5)}$. Entretanto, esse sintoma foi o menos relatado pelos sujeitos, nos dois grupos de pesquisa ${ }^{(3,16)}$.

Na identificação das estruturas afetadas pelos sintomas de ardência e secura bucal, a língua foi referida como o local mais atingido. Tal resultado concorda com a literatura pesquisada na SAB e com o envolvimento, também, de outras estruturas, mas em menor proporção ${ }^{(2-4,6)}$. Observou-se que o grupo xerostomia citou maior quantidade de estruturas envolvidas pelo sintoma de secura bucal, incluindo os lábios e a orofaringe ${ }^{(16,21,22)}$. Vale ressaltar que neste estudo, a estrutura orofaringe não foi relatada no G1, aparecendo com menor frequência na $\mathrm{SAB}^{(2,3)}$. Outra observação importante é que no quadro demonstrativo do número de estruturas afetadas pelo sintoma de secura bucal, visto anteriormente, a comparação entre os dois grupos já apontava tendência expressiva para o G2. Na identificação das estruturas, vimos que essa tendência foi confirmada.

A literatura refere que os indivíduos com queixa de ardência bucal e/ou secura bucal fazem uso dos medicamentos ${ }^{(4,6)}$, utilizam substitutos salivares, hidratantes labiais, gel, gomas de mascar, água com gotas de limão e estimulantes salivares ${ }^{(4,19,23)}$. No presente estudo, as estratégias mais significativas foram os alimentos, no $\mathrm{G} 1$ e os medicamentos, no $\mathrm{G} 2^{(2,6,10,11,14,23,25)}$. A utilização de bebidas, principalmente da água, aparece na literatura como uma estratégia comum aos dois grupos ${ }^{(11,14,15,22,29)}$. A utilização frequente somente da água é citada como capaz de produzir o efeito de alívio em pacientes com xerostomia ${ }^{(23)}$. Outras estratégias, como verificado na literatura ${ }^{(9-11,13,14)}$, também foram referidas. Nesta análise, parece importante ressaltar 
que, mesmo com o envolvimento de várias estruturas orais afetadas pelos sintomas de ardência e secura bucal, somente um sujeito do G1 mencionou "parar de falar" para alívio do sintoma de ardência, estratégia não citada no G2. Esse dado pode sugerir que os sintomas de ardência e/ou secura bucal, apesar das queixas dos sujeitos, não têm capacidade de interferir em sua comunicação ${ }^{(26,30)}$.

As queixas dos sujeitos com sintomas de ardência e/ou secura bucal são referidas na literatura ${ }^{(2,10,15,17,19,25,26)} \mathrm{e}$ com consequente impacto negativo na qualidade de vida $^{(16,24)}$, incluindo também o aspecto social ${ }^{(7,13)}$. No presente estudo, queixas como cansaço e força foram referidas por ambos os grupos, com destaque para o grupo de xerostomia. Estudos identificaram dificuldades nessas funções em sujeitos com secura bucal ${ }^{15,19,29)}$. Com relação à força citada na função da fala, poderia ser justificada pelo comprometimento dos articuladores da cavidade oral, principalmente da língua, levando a um maior esforço para a movimentação, por causa da lubrificação ineficiente. A literatura aponta que alterações que afetam a saliva, tanto em quantidade como em qualidade, poderão causar, dependendo da etiologia e grau de desconforto, prejuízos às funções de mastigação, deglutição e fala ${ }^{(15,21,26)}$.

Quanto à deglutição, pode-se inferir que a secura bucal denotaria necessidade de maior força, em presença da mucosa oral mais ressecada, em especial na região da orofaringe, referida pelo grupo com esse sintoma. Estudos verificaram alterações, principalmente nessa função, em indivíduos com Síndrome de Sjögren, sendo a disfagia referida como uma importante alteração associada à secura bucal ${ }^{(29)}$. A secura, em diversos graus de gravidade, poderia explicar a ocorrência da dificuldade na fala e deglutição desses indivíduos ${ }^{(15,19)}$.

Com relação à ocorrência de mudanças nos sintomas bucais, provocadas pelas funções orais, verificou-se, no G2, que a função de fala aumenta o sintoma de secura. A fala é uma função dinâmica que, associada à voz, necessita do mecanismo respiratório para sua realização, além da movimentação ritmada e sequencial de seus articuladores, língua e lábios, que podem demonstrar sinais de secura bucal ${ }^{(21)}$. Portanto, esperava-se, realmente, que sujeitos com sintoma de boca seca referissem aumento do sintoma após episódios de fala ${ }^{(27,29)}$.

Neste estudo, verificou-se baixa ocorrência de sujeitos que referiram aumento dos sintomas após a função de mastigação. A ingestão de alimentos, associada ao movimento mastigatório, favorece estímulos à salivação ${ }^{(12)}$, o que, provavelmente, provoca a sensação de alívio nesses sujeitos ${ }^{(10,16)}$. Além disso, muitos indivíduos mencionaram a ingestão de líquidos às refeições, possibilitando melhora na umidificação da mucosa oral ${ }^{(17,19,21,23,25)}$ para sujeitos com secura bucal e diminuição ou desaparecimento dos sintomas com ingestão de alimentos, para sujeitos com sintoma de ardência ${ }^{(6,11)}$.

Verificou-se que os grupos com sintomas bucais mostraram semelhança quanto às modificações das funções orais produzidas pelos sintomas bucais. Estudos demonstraram que a função de mastigação estaria comprometida em presença da secura bucal e que dificuldades na mastigação e deglutição são mais frequentes em avançado estágio de hipofunção da glândula salivar $^{(23,26,28)}$, sendo que apenas a hipossalivação não seria capaz de afetar o desempenho mastigatório ${ }^{(30)}$. Quanto à fala, apesar de 27,3\% dos sujeitos estudados com ardência referirem modificação, somente $4,5 \%$ relataram a estratégia "parar de falar" para alívio do sintoma. Um dado que pode associar-se a esse fato é o baixo percentual do sintoma de dor, que poderia, efetivamente, influenciar nas mudanças da mastigação, deglutição e fala. Desse modo, apesar das queixas relatadas pelos sujeitos, os sintomas de ardência e secura bucal parecem não ser capazes de causar grandes modificações nas funções orais, na ausência do sintoma de dor.

Quanto aos resultados relacionados às alterações de fala, estudos referem problemas com a fala, relatados pelos indivíduos com sintoma de secura bucal, que apresentam, em seu discurso, ou entre as sequências articulatórias, ruídos específicos, como "clique", identificados como estalidos ${ }^{(14-16,19,23)}$. Desse modo, pela diminuição da saliva, a língua aderida ao palato poderia levar à ocorrência de um "clique", perceptível no discurso de pacientes com síndrome de Sjögren, principalmente nos casos de extrema secura ${ }^{(18,29)}$. Tal ruído observado na fala não aparece associado à movimentação da mandíbula ${ }^{(30)}$, mas vinculado à movimentação da língua contra o palato ${ }^{(18,29)}$.

Pôde-se observar, pelos resultados deste estudo, a existência de queixas referidas por indivíduos que sofrem de sintomas orais crônicos, como ardência e secura bucal. Futuros estudos, com amostras maiores, poderão confirmar o perfil clínico oral desses indivíduos. A identificação e relevância das queixas, buscando maior conhecimento dessas características clínicas poderão auxiliar no direcionamento dos tratamentos que, mesmo não sendo curativos, possam minimizar o desconforto referido quanto às funções orais. Tais queixas, muitas vezes podem passar despercebidas e mascarar doenças que poderão influenciar futuramente e, de modo negativo, a qualidade de vida desses indivíduos. Desse modo, o papel do fonoaudiólogo que atua na área de diagnóstico e reabilitação da Motricidade Orofacial é o de verificar, por meio de avaliação criteriosa, queixas que possam associar-se e interferir nas funções de fala, mastigação e deglutição. O reconhecimento de diversos sinais e sintomas em cavidade oral propiciará o encaminhamento adequado, possibilitando o diagnóstico interdisciplinar precoce.

\section{CONCLUSÃO}

Das queixas envolvendo as funções orais, as de falar e deglutir com força foram as mais referidas pelos indivíduos do grupo xerostomia, com aumento do sintoma provocado pela função de fala. O sintoma de secura bucal, refletida pela presença de estalidos, interfere na fala e é constatada na grande maioria dos indivíduos com esse sintoma, seja ele principal ou associado à síndrome da ardência bucal. Não houve evidência 
de alteração fonética nos indivíduos com sintomas bucais, neste estudo. Os sintomas de ardência e secura bucal parecem não trazer prejuízos à função de fala em seu aspecto articulatório.

\section{REFERÊNCIAS}

1. Cherubini K, Maidana JD, Weigert KL, Figueiredo MA. Síndrome da ardência bucal: revisão de 100 casos. Rev Odonto Ciênc. 2005;20(48):109-13.

2. Rodríguez de Rivera Campillo ME, López López J, Chimenos Küstner E, Sabater Recolons MM. Estudio de una muestra de pacientes con síndrome de boca ardiente. Av Odontoestomatol. 2007;23(3):141-51.

3. Marino R, Torretta S, Capaccio P, Pignataro L, Spadari F. Different therapeutic strategies for burning mouth syndrome: preliminary data. J Oral Pathol Med. 2010;39(8):611-6.

4. Silvestre-Rangil J, Silvestre FJ, Tamarit-Santafé C, Bautista D. Burning mouth syndrome: correlation of treatment to clinical variables of the disease. Med Oral Patol Oral Cir Bucal. 2011;16(7):e890-4.

5. Gao J, Chen L, Zhou J, Peng J. A case-control study on etiological factors involved in patients with burning mouth syndrome. J Oral Pathol Med. 2009;38(1):24-8.

6. León Espinosa S, LópezJornet P, Frutos Ros R. Síndrome de boca ardiente. Eficacia de la aplicación tópica de capsaicina. Estudio piloto. Av Odontoestomatol. 2004;20(6):297-304.

7. Cavalcanti DR, Birman EG, Migliari DA, Silveira FRX. Burning mouth syndrome: clinical profile of Brazilian patients and oral carriage of Candida species. Braz Dent J. 2007;18(4):341-5.

8. Hershkovich O, Nagler RM. Biochemical analysis of saliva and taste acuity evaluation in patients with burning mouth syndrome, xerostomia and/or gustatory disturbances. Arch Oral Biol. 2004;49(7):515-22.

9. Gleber Netto FO, Diniz IMA, Grossmann SMC, Carmo MAV, Aguiar MCF. Síndrome da ardência bucal: uma revisão sobre aspectos clínicos, etiopatogenia e manejamento. Rev Cuba Estomatol. 2010;47(4):417-27.

10. Lauritano D, Calzavara D, Papagna R, Baldoni M, Bascones A. Evidencia del síndrome neuropático en un estudio neurofisiológico e inmunohistoquímico de las fibras nerviosas en pacientes con síndrome de boca ardiente. Av Odontoestomatol. 2003;19(2):81-94.

11. López Carriches C, Martínez-González JM, Gómes Font R, Leco Berrocal I, Donado Rodríguez M. Estudio clínico-epidemiológico sobre el síndrome de ardor bucal. Av Odontoestomatol. 2003;19(4):185-91.

12. Pedersen AM, Bardow A, Jensen SB, Nauntofte B. Saliva and gastrointestinal functions of taste, mastication, swallowing and digestion. Oral Dis. 2002;8(3):117-29.

13. Turner MD, Ship JA. Dry mouth and its effects on the oral health of elderly people. J Am Dent Assoc. 2007;138(suppl):15S-20S.
14. Glore RJ, Spiteri-Staines K, Paleri V. A patient with dry mouth. Clin Otolaryngol. 2009;34(4):358-63.

15. Cho MA, Ko JY, Kim YK, Kho HS. Salivary flow rate and clinical characteristics of patients with xerostomia according to its aetiology. J Oral Rehabil. 2010;37(3):185-93.

16. Gerdin EW, Einarson S, Jonsson M, Aronsson K, Johansson I. Impact of dry mouth conditions on oral health-related quality of life in older people. Gerodontology. 2005;22(4):219-26.

17. Rogus-Pulia NM, Logemann JA. Effects of reduced saliva production on swallowing in patients with Sjogren's syndrome. Dysphagia. 2011;26(3):295-303.

18. Kassan SS, Moutsopoulos HM. Clinical manifestations and early diagnosis of Sjögren syndrome. Arch Intern Med. 2004;164(12):1275-84.

19. Fox PC, Bowman SJ, Segal B, Vivino FB, Murukutla N, Choueiri $\mathrm{K}$ et al. Oral involvement in primary Sjögren syndrome. J Am Dent Assoc. 2008;139(12):1592-601.

20. Muñoz MGH, Castelão WCB, Saraiva FMD, Costa JCT, Queiroz MFOV. Síndrome de Sjögren primária: manifestações exócrinas e não exócrinas. Rev Bras Reumatol. 2004;44(2):129-38.

21. Farsi NMA. Signs of oral dryness in relation to salivary flow rate, ph, buffering capacity and dry mouth complaints. BMC Oral Health. 2007;7(15):1-6.

22. Villa A, Abati S. Risk factors and symptoms associated with xerostomia: a cross-sectional study. Aust Dent J. 2011;56(3):290-5.

23. Alpöz E, Güneri P, Onder G, Cankaya H, Kabasakal Y, Köse T. The efficacy of Xialine in patients with Sjögren's syndrome: a singleblind, cross-over study. Clin Oral Investig. 2008;12(2):165-72.

24. Suh KI, Lee JY, Chung JW, Kim YK, Kho HS. Relationship between salivary flow rate and clinical symptoms and behaviours in patients with dry mouth. J Oral Rehabil. 2007;34(10):739-44.

25. Pijpe J, Kalk WW, Bootsma H, Spijkervet FK, Kallenberg CG, Vissink A. Progression of salivary gland dysfunction in patients with Sjögren's Syndrome. Ann Rheum Dis. 2007;66(1):107-12.

26. Kaplan I, Zuk-Paz L, Wolff A. Association between salivary flow rates, oral symptoms, and oral mucosal status. Oral Surg Oral Med Oral Pathol Oral Radiol Endod. 2008;106(2):235-41.

27. Roh Jl, Kim HS, Kim AY. The effect of acute xerostomia on vocal function. Arch Otolaryngol Head Neck Surg. 2006;132(5):542-6.

28. Ishijima T, Koshino H, Hirai T, Takasaki H. The relationship between salivary secretion rate and masticatory efficiency. J Oral Rehabil. 2004;31(1):3-6.

29. Allec LDR, López XH, Porras JBA, Ramos RV, Pacheco del Valle JC, García AIP. Alteraciones de la voz, el habla y la deglución en pacientes con síndrome de Sjögren. Acta Otorrinolaringol Esp. 2011;62(4):255-64.

30. Gomes SGF, Cury, AADB, Garcia RCMR. Effect of hyposalivation on mastication and mandibular movements during speech. Braz Oral Res. 2011;25(4):351-6. 